\title{
Ultraminiature Antennas Combining Subwavelength Resonators and a Very-High- $\varepsilon$ Uniform Substrate: The Case of Lithium Niobate
}

\author{
Andriy E. Serebryannikov ${ }^{\circledR}$, Senior Member, IEEE, Mutlu Gokkavas, Tamara Funda Gundogdu, Vladimir Volski, \\ Guy A. E. Vandenbosch ${ }^{\circledR}$, Fellow, IEEE, Alexander Vasylchenko, and Ekmel Ozbay ${ }^{\circledR}$
}

\begin{abstract}
Combining the effects of subwavelength resonators and very-high-permittivity substrates enables a high extent of miniaturization, even for very simple, split-loop resonators. Here, we demonstrate how requirements to the substrate's permittivity are connected with the desired extent of miniaturization and why materials with a relative permittivity of $30<\varepsilon<100$, like lithium niobate, may offer a real possibility to miniaturize. For demonstration purposes, we designed, in line with this approach, an ultraminiature dual-band antenna to operate at 2.8 and $4.2 \mathrm{GHz}$. The antenna is fabricated using microfabrication techniques and studied experimentally. There is good agreement between the measurement and simulation results. The realized gain is about $-5 \mathrm{~dB}$ for the first resonance, at which the size of the substrateresonator block is $\lambda / 24$. The obtained results demonstrate the potential of the suggested approach, which is expected to be applicable to a very wide class of subwavelength resonators and a wide variety of substrates with high permittivity.
\end{abstract}

Index Terms - Lithium compounds, metamaterials, miniature antennas, multifrequency antennas.

\section{INTRODUCTION}

$\mathbf{T}$ HE demand for small antennas has been growing over the past decades, being stimulated by a variety of modern applications like implantable antennas, miniature wireless sensors, wearable sensor nodes, and multifunctional

Manuscript received November 22, 2019; revised January 30, 2020; accepted February 3, 2020. Date of publication February 27, 2020; date of current version July 7, 2020. This work was supported by the European Union's Horizon-2020 Research and Innovation Program through the Marie Skłodowska-Curie under Grant 708200. (Corresponding author: Andriy E. Serebryannikov.)

Andriy E. Serebryannikov is with the ESAT-TELEMIC Division, Katholieke Universiteit Leuven, 3001 Leuven, Belgium, on leave from the Faculty of Physics, Adam Mickiewicz University, 61-614 Poznań, Poland (e-mail: aserebry@esat.kuleuven.be).

Mutlu Gokkavas and Tamara Funda Gundogdu are with the Nanotechnology Research Center (NANOTAM), Bilkent University, 06800 Ankara, Turkey (e-mail: gokkavas@bilkent.edu.tr; tamara@bilkent.edu.tr).

Vladimir Volski and Guy A. E. Vandenbosch are with the ESAT-TELEMIC Division, Katholieke Universiteit Leuven, 3001 Leuven, Belgium (e-mail: vladimir.volski@esat.kuleuven.be; guy.vandenbosch@esat.kuleuven.be).

Alexander Vasylchenko is with Sofitto NV, 3000 Leuven, Belgium.

Ekmel Ozbay is with the Nanotechnology Research Center (NANOTAM), Bilkent University, 06800 Ankara, Turkey, also with the National Institute of Materials Science and Nanotechnology (UNAM), Bilkent University, 06800 Ankara, Turkey, also with the Department of Electrical Engineering, Bilkent University, 06800 Ankara, Turkey, and also with the Department of Physics, Bilkent University, 06800 Ankara, Turkey (e-mail: ozbay@ bilkent.edu.tr).

Color versions of one or more of the figures in this article are available online at http://ieeexplore.iee.org.

Digital Object Identifier 10.1109/TAP.2020.2975544 communication systems [1]. The development of small antennas for multiband operation has become a trend in mobile communications. A modern smartphone has several functions that need several antennas in one device, to be used for WiFi, GPS, cellular network bands, etc., while acceptable sizes are strongly restricted [2]. An alternative solution can be based on the use of one multiband antenna [3]-[7]. However, size issues can be critical in this case, too. The Internet-of-things (IoT) and healthcare applications are two more areas in which small antennas are in high demand [8], [9]. Although requirements for the number of bands and bandwidths can be mitigated for these applications compared to mobile communications, requirements for the maximal sizes also force one to use miniature antennas [10]-[13]. At the same time, the industry needs inexpensive small antennas that are well integrated with other electronics.

One of the approaches to antenna miniaturization is based on the use of subwavelength resonators (e.g., split-rings or split-loops) having the capability to confine energy at a subwavelength scale. Such antennas are often inspired by metamaterials [14]-[16]. A variety of antenna performances that represent a single unit cell of a metamaterial or metasurface, or a finite-extent portion of a metamaterial or metasurface, which consists of several unit cells, has been suggested (see [17]-[25]). On the other hand, dielectric resonator antennas, in which materials with a very high relative permittivity, e.g., up to 100, have been utilized, are well known [26], [27]. The recently proposed commercial dielectric antennas based on high-permittivity ceramics can have a very small electrical size [28], although the tradeoff between the output characteristics and packaging requirements does not always lead to well-suitable solutions.

Ceramic materials with a very high relative permittivity, i.e., $30<\varepsilon<100$, have been used as substrates in the miniaturized microstrip antennas with a conventional patch [29]-[31] and as a surrounding material in monopole (wire) antennas [32]. The approach based on a partial or nonuniform very-high- $\varepsilon$ filling of substrate has been proposed in [29], [33], and [34] for microstrip antennas with a conventional patch. However, it can be impractical for mass production. The natural approach to control the miniaturization capability is to combine the effects of subwavelength resonators and a very high permittivity of some of the used components in one antenna. However, the common effect of high- $\varepsilon$ components and 
subwavelength resonators on the achievable extent of miniaturization and characteristics of antennas has not yet been systematically studied.

Ferroelectrics represent a wide class of materials whose relative permittivity can exceed several hundreds and more have been utilized in various antennas to obtain dynamic tunability (by means of dc bias) and miniature design [35]-[38]. They have been used like either a substrate or local elements (chips), and in varactors. In particular, microstrip antennas on ferroelectric substrate [37], [38], coplanar-waveguide slot antennas containing thin ferroelectric films [35], and ferroelectric-air metamaterial inspired patch antennas [36] should be mentioned. While tunability is the basic function, the second function of ferroelectrics, i.e., miniaturization has also attracted a lot of attention. The work in [35] is probably only one example of miniaturization of the antenna of a quite complex configuration, which has a split-loop as one of the components. To the best of our knowledge, the common effect of the material properties of ferroelectrics and subwavelength resonators on miniaturization scenarios for monopole and patch antennas has been neither systematically studied nor experimentally demonstrated. It is worth noting that substrates with $\varepsilon>100$ can be impractical for antennas, while the range of $30<\varepsilon<100$ is still expected to be useful. Therefore, an air-ferroelectric powder may be needed rather than a uniform ferroelectric material.

A combination of subwavelength resonators and ferroelectric materials has been used in metamaterials, microstrip lines, and filters [39]-[42]. However, these results cannot be used to assess the capability of such combinations in radiation of future ultraminiature antennas. The same remains true of similar structures comprising non-ferroelectric very-high- $\varepsilon$ materials (see [43], [44]). One more approach has been proposed in [45], where a material with $\varepsilon=100$ (known as K-100) was placed into the resonator's gap (spacer) of a magnetic EZ antenna that yielded a fourfold decrease in the electrical size compared to the original antenna with a quartz spacer. However, neither a detailed analysis nor the experimental results were presented for this case.

Since the simplicity and low cost of fabrication are usually the key criteria for mass production, re-evaluation of the known affordable materials for the possible use in antenna miniaturization should be performed. In particular, lithium niobate $\left(\mathrm{LiNbO}_{3}\right)$, a non-ceramic electrooptic material, which is widely used in optical modulators [46]-[48], is a good candidate. $\mathrm{LiNbO}_{3}$ is a uniaxial anisotropic material with the components of the permittivity tensor, $\varepsilon_{\mathrm{xx}}=\varepsilon_{\mathrm{yy}}=43$ and $\varepsilon_{\mathrm{Zz}}=28$, at microwave frequencies [47]-[49]. Up to now, $\mathrm{LiNbO}_{3}$ has been widely used in electrooptical modulators, converters and sensors, and in bow-tie and patch antennas, which are integrated into electrooptical devices [49]-[53]. It has not yet been used in combination with subwavelength resonators, or just beyond electrooptics as a highpermittivity substrate for antenna miniaturization purposes. The only exception is [54], which presents the preliminary results on the combination of subwavelength resonators with a $\mathrm{LiNbO}_{3}$ substrate.
In this article, we study the possibility of combining the effects of subwavelength resonators and a-very-highpermittivity substrate $(30<\varepsilon<100)$ for antenna miniaturization. To demonstrate this approach, the results are presented for simple split-loops. The scaling of resonance frequencies is studied to find the connection between the desired extent of miniaturization and the number of obtainable subwavelength resonances, on the one hand, and between the desired extent of miniaturization and the required substrate's permittivity, on the other hand. Generally speaking, our study is about antennas whose properties are predominantly determined by a simultaneous effect of subwavelength resonators and substrates with a very high permittivity, but not about antennas based on the effect of one of them.

The focus is a dual-band ultraminiature antenna on a $\mathrm{LiNbO}_{3}$ substrate, which is designed to operate at 2.8 and $4.2 \mathrm{GHz}$. However, it can easily be redesigned to operate at WiFi, GPS, and other widely used standard frequencies. This antenna, with a size of the substrate-resonator block of only $\lambda / 24$ at the first resonance, was fabricated using microfabrication techniques and studied experimentally. Directions for further advancement are indicated for the used type of subwavelength resonators and substrate, including even a higher extent of miniaturization. All simulations were performed using the CST Studio Suite [55]. The main goal here is the proof of concept. Searching for an optimal antenna topology and a final prototype is beyond the scope. In contrast to [54], the effect of the substrate's permittivity on the scaling of the resonance frequencies and the extent of antenna miniaturization is discussed, angle-frequency dependence of electric field at a large distance from the antenna is obtained experimentally, the results of the far-field measurements performed in an anechoic chamber are presented, and possible modifications of antennas on a $\mathrm{LiNbO}_{3}$ substrate with a desired number of subwavelength bands are analyzed. In contrast to ferroelectrics based and some kinds of LTCC ceramics [56], no powder preparation is needed in the case of $\mathrm{LiNbO}_{3}$ to obtain $30<\varepsilon<100$, so it can be used without any preprocessing. In addition, our approach suggests a new application for $\mathrm{LiNbO}_{3}$, which is widely used in other areas of electrical engineering. The fabrication steps needed for the devices comprising this material are well developed, yielding a good basis for future mass production of the designed and similar antennas.

The questions that stimulated this article are as follows.

1) How efficient is a combination of subwavelength resonators and a high-permittivity substrate for antenna miniaturization?

2) $\mathrm{Can} \mathrm{LiNbO}_{3}$ be a suitable substrate for metamaterialinspired antennas?

3) Can the suggested approach be an alternative to commercial ceramic antennas?

This article is organized as follows. Section II presents the proposed antenna topology. In Section III, the theoretical results on scaling of the resonance frequencies are discussed. Section IV presents the experimental results and their comparison with simulations. Section V briefly illustrates some of the 


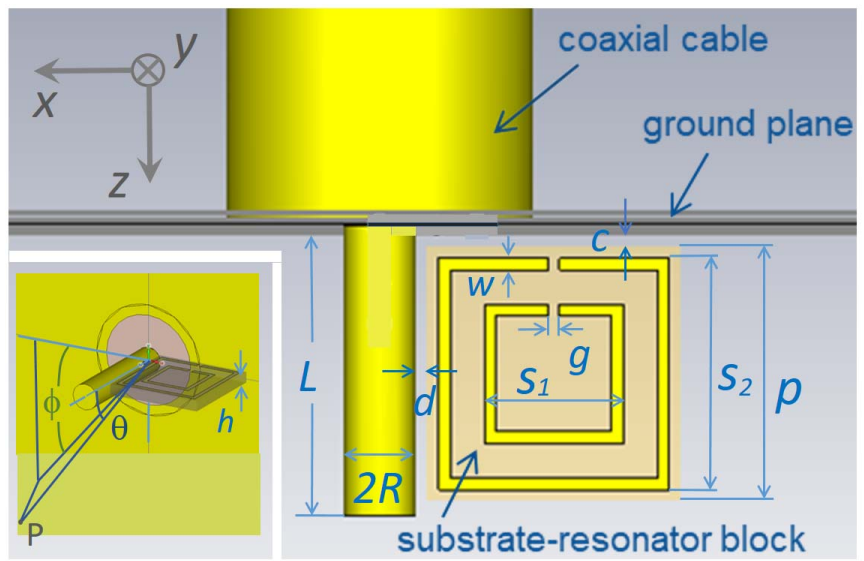

Fig. 1. Schematic of the antenna configuration with the basic set of splitloops (type A); inset shows the perspective view with angles $\theta$ and $\phi$, which describe the location of the observation point, $P ; \phi$ is measured in the $x y$ plane.

achievable combinations for resonance frequencies. Finally, Section VI formulates the conclusion.

\section{Proposed Antenna Topology}

To demonstrate the common effect of substrate permittivity and subwavelength resonator(s), we restrict the consideration here to such a dual-band configuration. It contains two subwavelength, square-shaped, split-loop resonators and the size-matched substrate, and is fed by a standard coaxial cable (see Fig. 1). Such antennas, coupled with an openended coaxial cable, are of interest in many applications where small external antennas are required. Each split-loop may create a separate band connected with its subwavelength resonance, even when being affected by the remaining loop, as well as by the coaxial wire and ground plane. It is worth noting that split-loop and split-ring resonators remain the most demanded subwavelength building elements, despite the fact that more advanced resonators with a smaller electrical size are known [43], [57], [58]. Embedding of split-loops (like in Fig. 1) and split rings has been used in some of the recently proposed antennas and metamaterials (see [17], [59]-[63]). Moreover, dual-band operation can be obtained in the subwavelength range even if using a single split-loop, due to the effect of the substrate. Indeed, as will be shown in Section III, some of the resonances, which are not subwavelength in the hypothetic substrate-free structure or a realistic structure with a low-permittivity substrate, can become subwavelength while increasing the substrate's permittivity. However, this approach to multiband operation in the subwavelength range has a disadvantage, because of the impossibility of controlling the spectral locations of two or more resonances simultaneously. That is why we use two split-loops, whose geometrical parameters and, thus, resonance frequencies can be chosen individually. In the studied antennas, radiation is expected to occur due to near-field coupling between the feed and subwavelength resonators, whereas there is no electrical contact between them. The general mechanism of near-field, electric, and magnetic coupling between the feed and subwavelength resonators has been widely used in practical antennas and frequently discussed in the antenna literature [16]-[18], [22], [60], [62].
The geometrical parameters for the configuration shown in Fig. 1 were chosen as follows: the side size of the inner and outer split-loops is $s_{1}=2.4 \mathrm{~mm}$ and $s_{2}=4 \mathrm{~mm}$, respectively; width of the gaps of the loops is $g=0.2 \mathrm{~mm}$; width of the strips, of which the loops are made, is $w=0.2 \mathrm{~mm}$; the side size of the substrate-resonator block is $p=4.4 \mathrm{~mm}$; and the substrate's thickness is $h=0.5 \mathrm{~mm}$. The loops are assumed to be made of copper, $18 \mu \mathrm{m}$ thick. The wire of the coaxial has a standard radius of $R=0.62 \mathrm{~mm}$ and length of $L=5.6 \mathrm{~mm}$. The distances between the ground plane and the substrateresonator block, $c$, and between the wire and the block, $d$, are initially chosen as 0.4 and $0.2 \mathrm{~mm}$, respectively. The angles $\theta$ and $\phi$ are shown in the inset in Fig. 1. When $\phi=0^{\circ}, \theta$ is varied in the $x z$ plane, i.e., in the split-loop resonator plane. In turn, $\theta$ is varied in $y z$ plane, i.e., perpendicular to the splitloop resonator plane, when $\phi=90^{\circ}$. Throughout this article, zero observation angle, $\theta=0^{\circ}$, corresponds to the case when $P$ is located on the $z$-axis.

\section{Scaling Resonance Frequencies}

The effect of antenna surroundings may be critical for the performance of miniature antennas. Clearly, being adjusted to the split-loops, the substrate can dramatically change the resonance frequencies. While the qualitative effect of the substrate's permittivity on the resonance frequencies is quite expected, its quantitative assessment invokes deep study. An extensive numerical study has been carried out for the antenna topology specified in Section II, with the aim to demonstrate the scaling of resonance frequencies and the possibility of control of the number of resonances at a fixed physical size of the substrate-resonator block, by means of variations in the substrate's relative permittivity, $\varepsilon_{\mathrm{S}}$, in the range extending from 1 to 60 . The resonances are assumed to correspond to the minima of $S_{11}$. The results are presented in Fig. 2. For the hypothetic substrate-free configuration with $\varepsilon_{\mathrm{s}}=1$, there is only one resonance in the frequency range of $f<9 \mathrm{GHz}$, which corresponds to $p<\lambda / 7.5$. By increasing $\varepsilon_{\mathrm{S}}$, one can shift the resonances, which include the ones being initially not (deeply) subwavelength, (deeper) into the subwavelength range. For example, two and more resonances can be obtained for $f<8.37 \mathrm{GHz}$ when $\varepsilon_{\mathrm{s}}>5.8$ (the resonance frequencies above the dash-dotted horizontal line in Fig. 2). Three resonances at $p<\lambda / 7.5(f<8.8 \mathrm{GHz})$ need a substrate with $\varepsilon_{\mathrm{S}}>43$ for the studied structure.

Scaling of resonance frequencies due to the effect exerted by dielectric constituents is probably best known for closed microwave cavities. In this case, the resonance frequencies are scaled according to the classical scaling rule, $f \propto A \varepsilon_{\mathrm{f}}^{-1 / 2}$, where $f$ is the frequency, $A$ is the scaling constant, and $\varepsilon_{\mathrm{f}}$ is the relative permittivity of the material that completely fills the cavity [64].

The specifics of open subwavelength resonators are that a large portion of the resonance field can be concentrated outside the resonator. This feature manifests itself in the fact that negative values larger than $-1 / 2$ and a different scaling constant typically appear in a scaling rule similar to the classical rule [65]. In other words, subwavelength resonances 


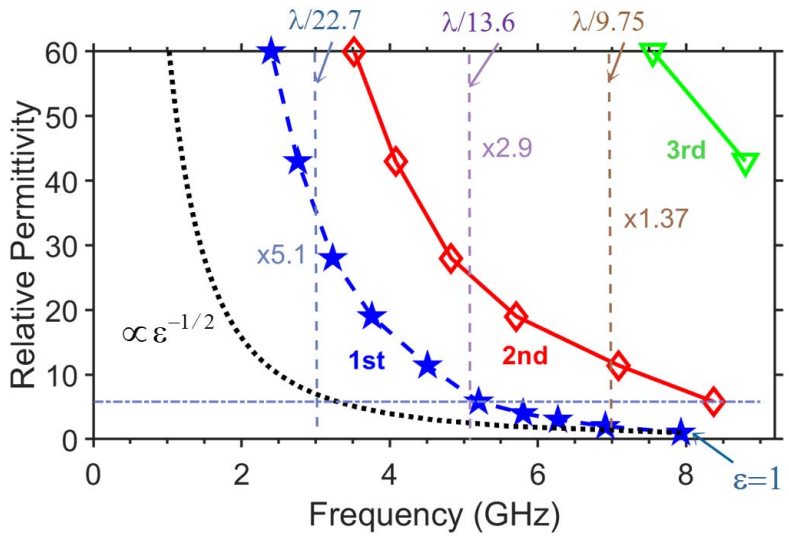

Fig. 2. Relative permittivity $\left(\varepsilon=\varepsilon_{\mathrm{S}}\right)$ versus resonance frequency for given geometrical parameters of the substrate-resonator block. Asterisks, rhombs, and triangles indicate the calculated values for the first, second, and third resonances, respectively. Dependence corresponding to the classical scaling rule $\left(\varepsilon=\varepsilon_{\mathrm{f}}\right)$ is shown for comparison by the thick dotted line.

in open structures (like the one in Fig. 1) can be sensitive to the permittivity variations in a different way than the resonances in a closed cavity. Variation of the lowest resonance frequency according to the classical scaling rule is shown in Fig. 2 by a dotted line, assuming that $A=f_{0}=7.93 \mathrm{GHz}$, which is equal to the lowest resonance frequency of the studied structure at $\varepsilon_{\mathrm{S}}=1$. Variations of the first, second, and third resonance frequencies are shown in Fig. 2, respectively, by asterisks, rhombs, and triangles. By comparing the dashed line corresponding to the first resonance and dependence shown by the dotted line in Fig. 2, one can see how requirements to the substrate material for the studied structure $\left(\varepsilon_{\mathrm{s}}\right)$ and to the filling material for the closed cavity $\left(\varepsilon_{\mathrm{f}}\right)$ are changed while varying the frequency. The difference between $\varepsilon_{\mathrm{s}}$ and $\varepsilon_{\mathrm{f}}$ increases while decreasing the frequency. So, a significantly larger value of $\varepsilon=\varepsilon_{\mathrm{S}}$ may be needed to obtain the same extent of miniaturization as in a closed cavity with $\varepsilon=\varepsilon_{\mathrm{f}}$. This explains why materials with a very high $\varepsilon$ may be demanded in open subwavelength structures.

For purposes of illustration, we consider three cases indicated by vertical lines in Fig. 2, which correspond to $p=$ $\lambda / 9.75, p=\lambda / 13.6$, and $p=\lambda / 22.7$. If $p=\lambda / 9.75$, it suggests a desirably small configuration, and one needs $\varepsilon_{\mathrm{s}}=1.37 \varepsilon_{\mathrm{f}}$. At $p=\lambda / 13.6$, one needs $\varepsilon_{\mathrm{s}}=2.9 \varepsilon_{\mathrm{f}}$. Thus, the higher the extent of miniaturization that is required, the larger the difference in permittivity between the closed cavity $\left(\varepsilon_{\mathrm{f}}\right)$ and the open resonator $\left(\varepsilon_{\mathrm{S}}\right)$ should be. However, if an ultraminiature configuration is needed, the difference between $\varepsilon_{\mathrm{f}}$ and $\varepsilon_{\mathrm{S}}$ is even larger, like in the case of $p=\lambda / 22.7$, where it is nearly fivefold. For the closed cavity, such miniaturization would need a high $\varepsilon_{\mathrm{f}}$, i.e., $\varepsilon_{\mathrm{f}}=7$, which is still a conventional substrate. But this is not the case for the dual split-loop structure, for which $\varepsilon_{\mathrm{s}}=35$ is required. A large slope of the dependence of the relative permittivity on frequency is observed in Fig. 2 not only for the case of the split-loop structure but also for the closed-cavity case, but at smaller frequencies. As seen from the comparison of the dashed and dotted lines, it is located, respectively, below 5 and $2 \mathrm{GHz}$. This is a reason why $\varepsilon_{\mathrm{s}}$ being significantly larger than $\varepsilon_{\mathrm{f}}$ in the closed cavity is required in the case of $p=\lambda / 22.7$.

The requirements to $\varepsilon_{\mathrm{S}}$ depend on the resonator type and required size and can be mitigated for more complex subwavelength resonators, which may themselves enable a higher extent of miniaturization (see [43], [57], [58]). Note that the extent of miniaturization achieved in [35] and [36] with the help of ferroelectric materials is weaker than in the proposed design, although it is connected to the way of use of these materials rather than to their capability in miniaturization.

\section{EXPERIMENTS}

The antenna's far-field components always contain the contributions from the two distinctive antenna elements: the substrate-resonator block and the wire of the cable. These contributions are summarized in Table I, in terms of the main field components. Both the split-loop resonators and the coaxial wire may contribute almost equally to the resulting radiation of the antenna. The contribution of each antenna element can be either co-polar or cross-polar, depending on the selected far-field component and the cutting plane. The contribution of the wire is expected to be very similar to that of a conventional dipole. Experiments have been conducted using two setups, which are described below.

\section{A. Fabrication and First Measurement Setup}

From the results presented in Section III, it is clear why substrates with $\varepsilon_{\mathrm{s}}>35$ are needed for ultraminiature configurations with $p<\lambda / 20$. From this viewpoint, the interest in $\mathrm{LiNbO}_{3}$ as a perspective substrate enabling a high extent of miniaturization can be understood.

The dual split-loop structure on the $\mathrm{LiNbO}_{3}$ substrate has been fabricated at the Bilkent University. The 3-in $x$-cut $\mathrm{LiNbO}_{3}$ wafers were coated with the $100 \AA \mathrm{Ti} / 2000 \AA \mathrm{Au}$ seed layer at high vacuum in an e-beam evaporator system. Photolithographic pattern definition was performed using $16 \mu \mathrm{m}$ thick KMPR 1025 photoresist. The wafers were coated with $3 \mu \mathrm{m}$ thick Au in a commercially available electroplating solution (Transene TSG-250). The seed layer was removed by wet etching in aqua regia/Ti etchant $\left(\mathrm{H}_{2} \mathrm{O}_{2}: \mathrm{HF}\right)$ to finalize the wafer scale antenna fabrication process. The samples were separated using an automated dicing saw. A change in material of the loops and deviation of metal thickness compared with the designed structure described in Section II was necessary in order to maintain compatibility with the standard fabrication processing recipes. Although examples are known where a metal choice for an antenna significantly affects the output characteristics [12], in our case they are mainly sensitive to the strip thickness rather than to the material choice, as confirmed by the obtained simulation results (not shown).

The photographs of the fabricated substrate-resonator block and the assembled structure are presented in Fig. 3(a) and (b), respectively. The first series of the experiments has been 
TABLE I

Main Field Components in Different Antenna Planes

\begin{tabular}{ccc}
\hline \hline & Resonator & Wire \\
\hline $\mathrm{E}_{\theta}, \phi=0^{0}$ & Copolar & Copolar \\
\hline $\mathrm{E}_{\phi}, \phi=0^{0}$ & Crosspolar & Crosspolar \\
\hline $\mathrm{E}_{\phi}, \phi=90^{0}$ & Copolar & Crosspolar \\
\hline $\mathrm{E}_{\theta}, \phi=90^{0}$ & Crosspolar & Copolar \\
\hline \hline
\end{tabular}

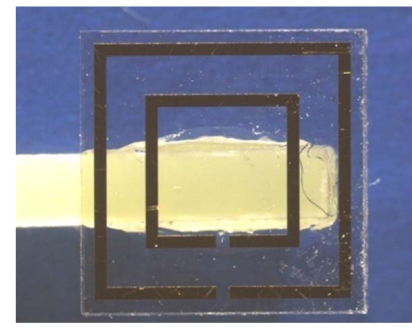

(a)

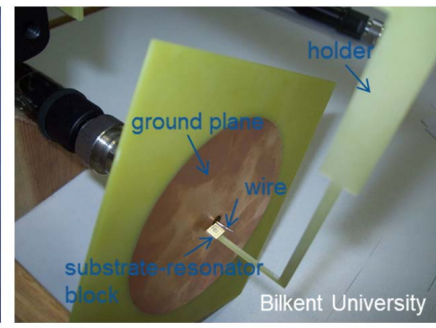

(b)
Fig. 3. Photographs of (a) fabricated substrate-resonator block with a part of the holder and (b) assembled structure for the first measurement setup.

performed at the Bilkent University. A holder made of a low$\varepsilon$ material has been used to mechanically fix the substrateresonator block with respect to the coaxial wire [see Fig. 3(b)]. It has been attached to the back side of the substrate-resonator block, as seen in Fig. 3, so that its effect on the resonance frequencies is insignificant (note the high permittivity contrast between $\mathrm{LiNbO}_{3}$ and the holder material and transparency of $\mathrm{LiNbO}_{3}$ at the visible frequencies).

Checking the principal possibility of radiation from the designed ultraminiature structure at its resonance frequencies retrieved from the $S_{11}$ results and obtaining a full map of the electric field in the frequency-observation angle plane at a large distance from the designed antenna are the main goals of the measurements performed using the first setup. An HP8510C network analyzer was used to measure $S_{11}$. The measured and calculated $S_{11}$ are presented in Fig. 4. There is very good coincidence in the spectral location of the dip of $\mathrm{S}_{11}$ for the first resonance, while the difference for the second resonance is about $2.5 \%$. The measured minima are deeper and show lower $Q$ than the calculated ones. Among the possible causes of the observed discrepancy, one should mention the losses in $\mathrm{LiNbO}_{3}$, which are approximately taken into account in simulations ( $\tan \delta=0.005)$, compared to [66], and deviations of the substrate-resonator block from the desired location with respect to the wire and specifics of its mechanical fixing. However, the full analysis of this discrepancy is beyond the scope of this article, which focuses on demonstration of the general concept rather than a particular performance. According to the measured data, $Q=51$ and 67 for the first and the second resonance, respectively. The results presented in the insets in Fig. 4, which show the distribution of the surface currents, give evidence of a weak mutual coupling between the split-loops and the dominant contribution of the outer split-loop to the first resonance and the inner split-loop to the second one.

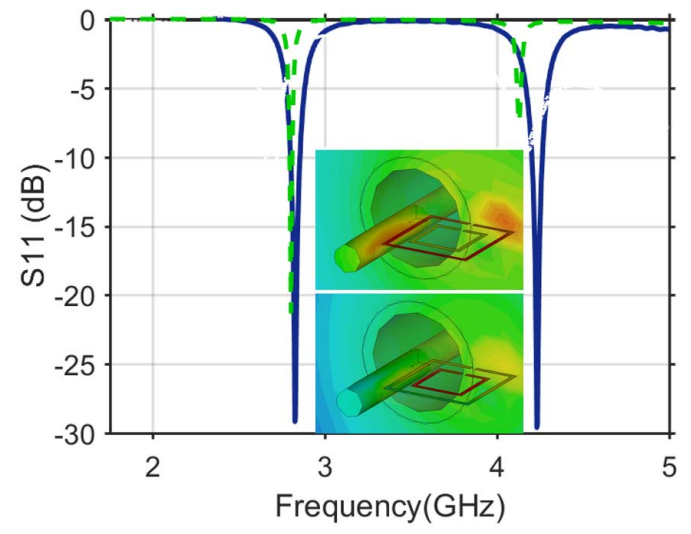

Fig. 4. Measured (solid line) and simulated (dashed line) $\mathrm{S}_{11}$, first setup; simulated surface currents are shown for the first resonance (upper inset) and second resonance (lower inset); most dark red color corresponds to the strongest current, and color change to green and then blue corresponds to the current weakening.

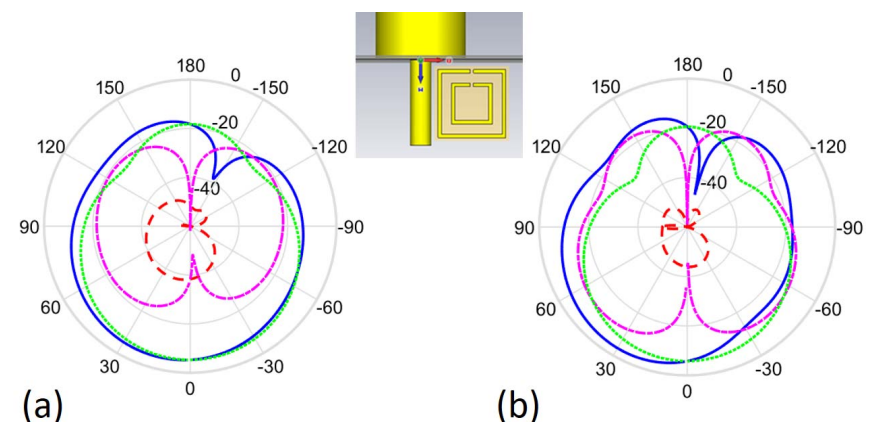

Fig. 5. Simulated results for realized gain, $G_{r}$ (in $\mathrm{dB}$ ) versus $\theta$ for (a) first $(f=2.8 \mathrm{GHz})$ and (b) second $(f=4.126 \mathrm{GHz})$ resonance; magenta lines- $\mathrm{E}_{\theta}, \phi=90^{\circ}$, green lines- $\mathrm{E}_{\phi}, \phi=90^{\circ}$, red lines- $\mathrm{E}_{\phi}, \phi=0^{\circ}$, and blue lines- $\mathrm{E}_{\theta}, \phi=0^{\circ}$.

For small antennas, the following estimate can be used for the gain, $G$ [67]:

$$
G=(k a)^{2}+2 k a
$$

where $k$ is the free-space wavenumber and $a$ is the radius of a sphere completely containing the antenna. The expected values of $G_{r}$ are $-0.56 \mathrm{~dB}$ when the wire is taken into account and $-2.14 \mathrm{~dB}$ when it is not taken into account in the $a$-value, for the first resonance. For the second resonance, it is 1.44 and $-0.5 \mathrm{~dB}$, respectively. The mirror image of the antenna with respect to the ground plane was taken into account in the value of $a$ to obtain these estimates. Smaller values of $G$ are obtained from (1) when the image is not taken into account.

Next, based on the simulation results, we estimated the far-field characteristics and the shape of the radiation pattern (see Fig. 5). In the resonator (split-loop) plane, the maximum is rotated outward from the substrate-resonator block. In the plane perpendicular to the split-loops, the pattern is almost symmetric. The radiation efficiency, $\eta_{\mathrm{rad}}$, is -11.3 and $-10 \mathrm{~dB}$, and $G_{r}$, is -4.9 and $-3.4 \mathrm{~dB}$, for the first and the second resonance, respectively. These values are acceptably lower than the predictions based on (1). 

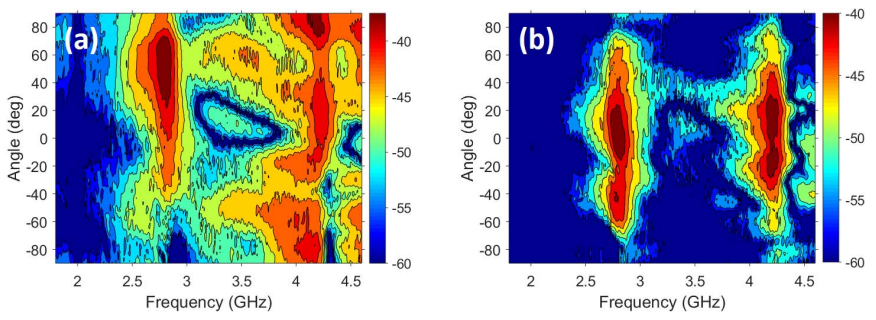

Fig. 6. Measured electric field (in dB) as a function of frequency and observation angle in (a) $x z$ plane $\left(\phi=0^{\circ}, \mathrm{E}_{\theta}\right.$ component) and (b) $y z$ plane $\left(\phi=90^{\circ}, \mathrm{E}_{\phi}\right.$ component).

Contributions of the substrate-resonator block and the wire of the coaxial are recognizable.

To prove the principal possibility of radiation at the resonance frequencies predicted by the $S_{11}$ data, a scanning has been carried out by varying the observation angle, $\theta$, while $\phi$ is equal to either $0^{\circ}$ or $90^{\circ}$. The setup differs from the one used to obtain the results in Fig. 4, only in that the standard receiver horn antenna has been added. A distance of $100 \mathrm{~cm}$ between the receiver and the antenna under test has been chosen. The obtained results are presented in Fig. 6. They indicate the existence of radiation at the minima of $S_{11}$. The main features expected from the simulation results, i.e., shift of the maximum from $\theta=0^{\circ}$ toward larger $\theta$ when scanning in the plane of split-loop resonators $\left(\phi=0^{\circ}\right)$, and symmetry with respect to $\theta=0^{\circ}$ when scanning perpendicularly to the resonators $\left(\phi=90^{\circ}\right)$, are observed in the experimental data. However, the maxima of the electric field in Fig. 6(a) correspond to larger values of the angle compared to the simulated $G_{r}$ in Fig. 5.

\section{B. Second Measurement Setup}

The second series of measurements has been conducted at the Katholieke Universiteit Leuven with the focus on the far-field characteristics. The measurements were performed inside an anechoic chamber using a dedicated setup based on an HP8530 microwave receiver. The size of the anechoic chamber is about $9.76 \mathrm{~m} \times 3.66 \mathrm{~m} \times 3.2 \mathrm{~m}$. The distance between two antennas was about $7 \mathrm{~m}$. An EMCO 3115 wide band horn was mounted at the $\mathrm{Tx}$ site. The antenna under test was fixed on a special holder on the Rx positioner. The Rx positioner performed an automatic scan in the azimuthal plane. Then the antenna polarization was manually updated for additional scans. The antenna matching measurement was performed using an H8510 vector network analyzer.

First, the goal was to achieve a better coincidence between the measured and simulated far-field results. In order to provide a reliable mechanical fixing of the substrate-resonator block with respect to the coaxial wire, a volumetric holder made of plexiglass $(\varepsilon=2.55$ and $\tan \delta=0.0039)$ has been used [see Fig. 7(a)]. The block was placed inside the holder. In order to keep the assembling simple, the wire length is matched with the size of the volumetric holder ( $L=11 \mathrm{~mm}$ ), so that its effect on the resulting radiation should start to appear at smaller frequencies than for the first
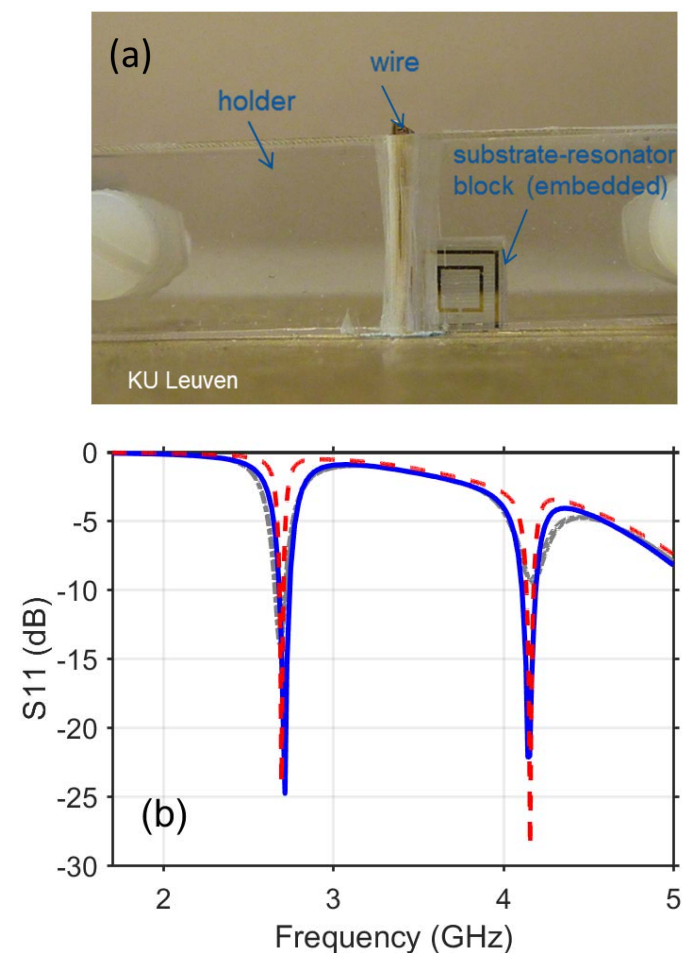

Fig. 7. (a) Photograph of the assembled structure for the second measurement setup. (b) Measured (solid line) and simulated (dashed lines) $\mathrm{S}_{11}$. In simulations, $c=0.4 \mathrm{~mm}$ and $d=0.8 \mathrm{~mm}$.

setup in Fig. 3(b). It is noteworthy that the proof of concept is the main purpose of this article, while optimal assembly is beyond its scope. Thus, the topology with the external holder is used here only for the purposes of the feasibility study. For future practical applications and mass production, the antenna can be fixed using an electrically conductive silver epoxy or similar glue, rather than such a holder.

Fig. 7(b) presents the results of simulation and measurement for $S_{11}$. The coincidence in the spectral location of the resonances is very good, with a difference of less than $1.5 \%$. The difference in the $Q$-factor is similar to that in the first setup. According to the measurement results, $Q=43$ and 50 for the first and the second resonance, respectively, which is a bit lower than that in the first setup. As a consequence of the extension of the central wire up to the holder edge, the contribution of the current induced on it to the antenna far-field pattern should be increased and interfere with the contribution from the split-loop resonators. The wire current generates an omnidirectional dipole-like pattern. This may result in a specific mix of different contributions depending on the far-field plane and the polarization, as shown in Table I. The contribution of the resonator only can be easily estimated by considering the $\mathrm{E}_{\phi}$ component at $\phi=90^{\circ}$, since the wire contribution is negligible in this case. Consulting Table I, such pattern can be expected to occur for the $\mathrm{E}_{\theta}$ component at $\phi=90^{\circ}$, where the wire contribution is dominant (co-polar), while the contribution of the substrate-resonator block is negligible because it is cross-polar. 


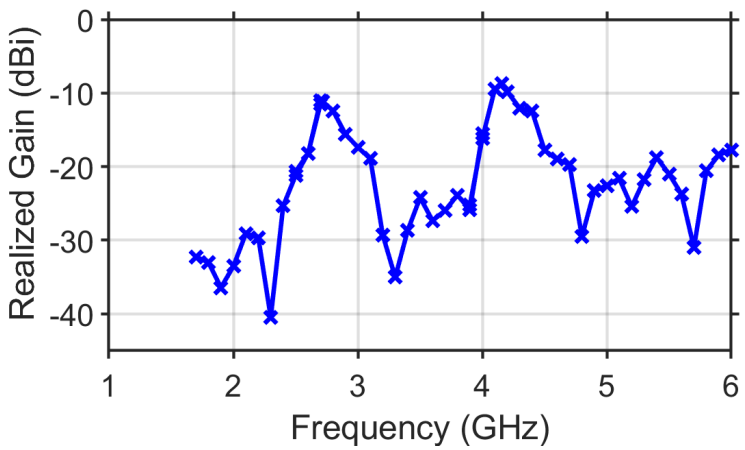

Fig. 8. Realized gain versus frequency at $\theta=0^{\circ}$ and $\phi=0^{\circ}, \mathrm{E}_{\theta}$ component (measurements).
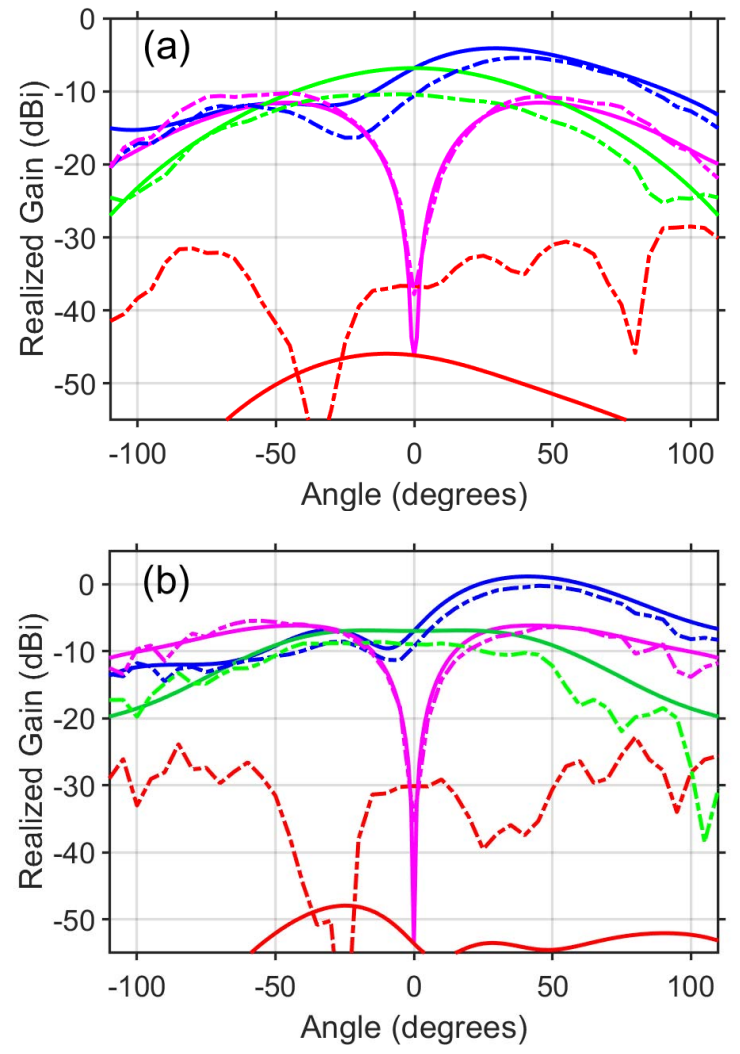

Fig. 9. Realized gain for (a) first and (b) second resonances. Solid lines-simulations. Dashed-dotted lines-measurement. Magenta lines- $\mathrm{E}_{\theta}$, $\phi=90^{\circ}$. Green lines- $\mathrm{E}_{\phi}, \phi=90^{\circ}$. Red lines- $\mathrm{E}_{\phi}, \phi=0^{\circ}$. Blue lines- $\mathrm{E}_{\theta}$, $\phi=0^{\circ}$.

The simulations predict $\eta_{\mathrm{rad}}=-10.5 \mathrm{~dB}$ for the first resonance and $-6 \mathrm{~dB}$ for the second resonance, while $G_{r}=-4.1$ and $1.23 \mathrm{~dB}$, respectively. Thus, the values of $\eta_{\mathrm{rad}}$ and $G_{r}$ are quite close for the first and second setups in the case of the first resonance. In the case of the second resonance, they differ stronger due to a stronger effect of the wire being longer for the second setup.

The antenna gain was measured using the gain transfer method with three reference horn antennas (Narda 645, Narda 644, and Narda 643) covering the whole frequency band of interest. Fig. 8 shows the measured gain versus frequency in the normal direction $\left(\theta=0^{\circ}\right)$. Two maxima are observed,

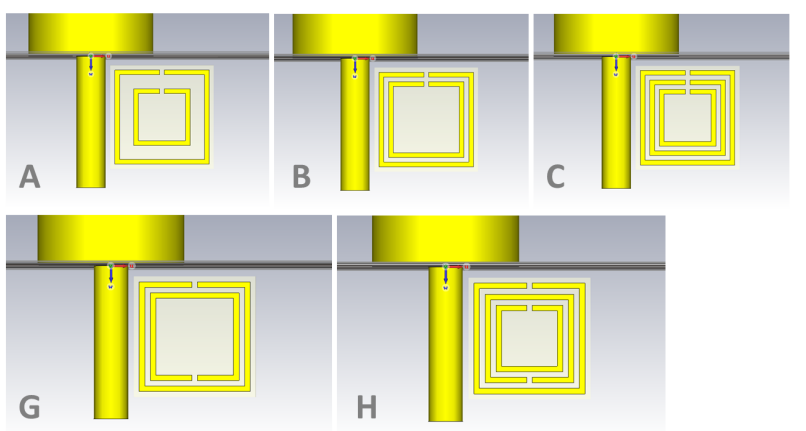

Fig. 10. Upper line, from left to right: resonator types A, B, and C; lower line, from left to right: types $\mathrm{G}$ and $\mathrm{H}$.

with $G_{r}=-11$ and $-8.7 \mathrm{~dB}$, whose spectral locations well coincide with the minima of $S_{11}$ in Fig. $7(b)$. Note that the maximum radiation is expected at $|\theta|>0$, so these maxima of $G_{r}$ are not the absolute ones.

A comparison of the simulation and experiment results for $G_{r}$ versus observation angle, $\theta$, is presented in Fig. 9 for the first and second resonances. In spite of $G_{r}$ being smaller in the measured data, the coincidence for the $\mathrm{E}_{\theta}$ component at $\phi=0^{\circ}$ is good for both the resonances, as well as for the $\mathrm{E}_{\phi}$ component at $\phi=90^{\circ}$. Coincidence in the angular location of $\max G_{r}$ is better for the second resonance [see Fig. 9(b)]. In the measured data in Fig. 9, $\max G_{r}=-5.4$ and $-0.2 \mathrm{~dB}$ for the $\mathrm{E}_{\theta}$ component scanned in the split-loop resonators plane, i.e., in the $x z$ plane $\left(\phi=0^{\circ}\right)$, for the first and the second resonances, respectively. Good coincidence is also achieved for the $\mathrm{E}_{\theta}$ component at $\phi=90^{\circ}$, which corresponds to the wire radiation, while scanning is performed in the plane perpendicular to the split-loops, i.e., in the $y z$ plane. The presented results show that the designed ultraminiature antenna radiates at the resonance frequencies of the substrate-resonator block, and all the basic features in the far-field behavior that are predicted by simulations are observed in the experiment.

\section{OTHER Modifications}

In this section, we demonstrate that the different numbers of the subwavelength resonances with different spectral distances between them can be obtained and, thus, demonstrate the generality of the concept. Let us discuss which modifications of the resonance frequencies are achievable, compared to the basic configuration, i.e., at the same geometrical sizes of the substrate-resonator block and the individual split-loops as in Section II. We add one more (middle) loop equidistant to the two loops in Fig. 1, i.e., with the side size of $s_{3}=\left(s_{1}+s_{2}\right) / 2$, while the split-loop of smaller side size $\left(s_{1}\right)$ is either retained or removed. The location of some of the remaining loops can be kept, while some others are rotated by $180^{\circ}$, i.e., they have opposite orientation of the loop gaps with respect to the ground plane.

As a result, we obtained several structures with a different number of split-loops. The schematics of these structures are presented in Fig. 10. The main geometrical parameters are given in Section II. The results are summarized in Table II. 
TABLE II

EFFECT OF Number, SizE, AND ORIENTATION OF SPLiT-Loops ON RESONANCE FREQUENCY BELOW $10 \mathrm{GHz}$

\begin{tabular}{cccc}
\hline \hline $\begin{array}{c}\text { Resonator } \\
\text { Type }\end{array}$ & $\begin{array}{c}\text { First } \\
\text { Resonance } \\
(\mathrm{GHz})\end{array}$ & $\begin{array}{c}\text { Second } \\
\text { Resonance } \\
(\mathrm{GHz})\end{array}$ & $\begin{array}{c}\text { Third } \\
\text { Resonance } \\
(\mathrm{GHz})\end{array}$ \\
\hline A & 2.84 & 4.18 & 9.06 \\
B & 2.61 & 3.26 & \\
C & 2.61 & 3.09 & 4.08 \\
D & 3.02 & & \\
E & 3.47 & & \\
F & 4.41 & 4.34 & 8.9 \\
G & 1.78 & 3.27 & 5.15 \\
H & 1.7 & & \\
\hline \hline
\end{tabular}

They show that the number of resonances in the subwavelength range, the lowest resonance frequency, and spectral distance between the resonance frequencies can be easily controlled, while the maximal sizes of the substrate-resonator block are kept. The resonator type denoted by A is exactly the same as that introduced in Section II, i.e., it uses $18 \mu \mathrm{m}$ thick copper strips. Therefore, resonance frequencies may slightly differ from those in Section IV.

By increasing the inner loop's side size, one can downshift the first and second resonance frequencies by $8.1 \%$ and $22 \%$, respectively (compare resonator types $\mathrm{A}$ and $\mathrm{B}$ ). Indeed, by increasing the side size of the inner loop for type B from $s_{1}$ to $s_{3}$, we increase the effective inductivity that decreases the second resonance frequency. Moreover, additional capacitance is introduced by approaching the outer loop by the inner one, which also contributes to the frequency decrease. Besides, the capacitances between the substrate-resonator block and coaxial wire and between the block and ground plane can affect at least the first resonance frequency.

If we take the type $\mathrm{A}$ and modify it by placing the middle loop, which is the same as the inner loop in type B, between the outer and inner loops of the radii $r_{2}$ and $r_{1}$, respectively, we obtain the structure of type C. In this case, three subwavelength resonances are achieved at $f<4.2 \mathrm{GHz}$ ( $p=\lambda / 16.2$ at $4.2 \mathrm{GHz}$ ). It is interesting that the lowest resonance frequency is almost insensitive to the inner loop of the radius $r_{1}$ for type $\mathrm{C}$, compared with type $\mathrm{B}$. In all the three cases, i.e., A, B, and $\mathrm{C}$, each of the split-loops provides a dominant contribution to one of the resonances. Thus, each loop is working more or less separately from the others, rather than contributing to an "effective-medium" resonance, as in [43]. The numerical results obtained for the surface currents (not shown) confirm this conclusion. To compare, the results are also presented in Table II for the split-loop configurations $\mathrm{D}, \mathrm{E}$, and F, which are obtained by keeping only one of the three loops (and removing two others) in configuration $\mathrm{C}$, i.e., the outer, the middle, and the inner loop, respectively. One can see there a single resonance at $f<10 \mathrm{GHz}$.

If one needs a larger difference between the lowest (first) and second resonance frequencies and, furthermore, a higher extent of miniaturization, a configuration with two split-loops can be used, which is similar to that of type B, but its inner loop's gap is rotated by $180^{\circ}$ (see Fig. 10 for type G and the corresponding data in Table II). Then, the first resonance frequency, $f=1.78 \mathrm{GHz}$, corresponds to $p=\lambda / 38$, whereas the second-to-first resonance frequency ratio is 2.44 . Finally, by making a similar modification for type $\mathrm{C}$, i.e., by rotating the middle loop by $180^{\circ}$, one obtains three resonance frequencies between 1.7 and $5.15 \mathrm{GHz}$, so that there is a threefold difference between the first and third resonance frequencies; see the data for type $\mathrm{H}$ in Table II and Fig. 10. The first resonance occurring at $1.7 \mathrm{GHz}$ corresponds to $\lambda / 40$. This is the highest extent of miniaturization in the structures studied in this article. The electrical size can also be decreased using other, also more complex resonator shapes than a split-loop, e.g., like those in [23], [25], [43], [57], [58], and [62]. This is a prospective topic for future studies.

\section{CONCLUSION}

To summarize, we demonstrated how the miniaturizing effects of subwavelength split-loop resonators and a veryhigh-permittivity substrate can be efficiently combined in a dual-band ultraminiature antenna with the operating bands at 2.8 and $4.2 \mathrm{GHz}$. Scaling of the resonance frequencies can be obtained while replacing one substrate with another and keeping all geometrical sizes constant. The obtained results show that the achievable extent of miniaturization and the number of subwavelength resonances may strongly depend on the chosen substrate material. They justify the necessity of using substrates with a permittivity of $\varepsilon_{\mathrm{S}}>25$ when a size of substrate-resonator block that is smaller than $\lambda / 20$ is needed.

The experimental demonstration was focused on an ultraminiature dual-band antenna on a $\mathrm{LiNbO}_{3}$ substrate, with $\lambda / 24$ side size of the substrate-resonator block at the lower operating frequency. The antenna on the $\mathrm{LiNbO}_{3}$ substrate was fabricated using standard microfabrication techniques. Good agreement between the experiment and simulation results was observed. The realized gain being about $-5 \mathrm{~dB}$ was obtained, which is acceptable for such a small size. For practical applications, various ways of integration of our antenna are possible, including an open-end coaxial input. It is low-profile and lowcost. In current research laboratory conditions, the cost of one antenna can be less than 1 USD, which makes it a suitable candidate for mass production.

A higher extent of miniaturization, e.g., with a $\lambda / 40$ side size, is possible at a proper choice of the location of the individual split-loops with respect to each other, without changing the maximal physical size of the structure. Moreover, triple-band operation can be obtained in the subwavelength regime at the same size. The obtained results indicate a new direction for $\mathrm{LiNbO}_{3}$ application, as far as its properties as a high-permittivity substrate for antenna miniaturization have not yet been exploited. A proper selection of the geometrical parameters of the split-loops allows to readjust the resonances to the frequency bands, which are widely used in practice, i.e., WLAN, upper band Wi-Fi, GPS, WiMaX, and 3G/4G cellular network bands. Various high-permittivity materials and advanced shapes of subwavelength resonators are planned to be studied in the future. 


\section{ACKNOWLEDGMENT}

A. E. Serebryannikov would like to thank Dr. K. B. Alici for fruitful discussions.

\section{REFERENCES}

[1] P. S. Hall and Y. Hao, Antennas and Propagation for Body-Centric Wireless Communications. Norwood, MA, USA: Artech House, 2006.

[2] P. Vainikainen et al., "More than 20 antenna elements in future mobile phones, threat or opportunity?" in Proc. 3rd Eur. Conf. Ant. Propag., Berlin, Germany, Mar. 2009, pp. 23-27.

[3] J.-W. Lian, Y.-L. Ban, Y.-L. Yang, L.-W. Zhang, C.-Y.-D. Sim, and K. Kang, "Hybrid multi-mode narrow-frame antenna for WWAN/LTE metal-rimmed smartphone applications," IEEE Access, vol. 4, pp. 3991-3998, 2016.

[4] H. Wang et al., "Small-size reconfigurable loop antenna for mobile phone applications," IEEE Access, vol. 4, pp. 5179-5186, 2016.

[5] M. Zheng, H. Wang, and Y. Hao, "Internal hexa-band folded monopole/dipole/loop antenna with four resonances for mobile device," IEEE Trans. Antennas Propag., vol. 60, no. 6, pp. 2880-2885, Jun. 2012.

[6] D. Wu, S. W. Cheung, and T. I. Yuk, "A compact and low-profile loop antenna with multiband operation for ultra-thin smartphones," IEEE Trans. Antennas Propag., vol. 63, no. 6, pp. 2745-2750, Jun. 2015.

[7] H. Xu et al., "A compact and low-profile loop antenna with six resonant modes for LTE smartphone," IEEE Trans. Antennas Propag., vol. 64, no. 9, pp. 3743-3751, Sep. 2016.

[8] S. M. R. Islam, D. Kwak, M. H. Kabir, M. Hossain, and K.-S. Kwak, "The Internet of Things for health care: A comprehensive survey," IEEE Access, vol. 3, pp. 678-708, 2015.

[9] D. Evans, "The Internet of Things: How the next evolution of the Internet is changing everything," Cisco, San Jose, CA, USA, White Paper, 2011. [Online]. Available: https://www.cisco.com/ c/dam/en_us/about/ac79/docs/innov/IoT_IBSG_0411FINAL.pdf

[10] L. Lizzi, F. Ferrero, P. Monin, C. Danchesi, and S. Boudaud, "Design of miniature antennas for IoT applications," in Proc. IEEE 6th Int. Conf. Commun. Electron. (ICCE), Ha Long, Vietnam, Jul. 2016, doi: 10.1109/CCE.2016.7562642.

[11] M. A. Khan, M. A. ul Haq, and S. ur Rehman, "A practical miniature antenna design for future Internet of Things enabled smart devices," in Proc. 10th Int. Conf. Signal Process. Commun. Syst. (ICSPCS), Golden Coast, QLD, Australia, Dec. 2016, pp. 1-6, doi: 10.1109/ICSPCS 2016.7843339.

[12] L. Lizzi and F. Ferrero, "Use of ultra-narrow band miniature antennas for Internet-of-Things applications," Electron. Lett., vol. 51, no. 24, pp. 1964-1966, Nov. 2015.

[13] M. W. A. Khan, E. Moradi, L. Sydänheimo, T. Björninen, Y. Rahmat-Samii, and L. Ukkonen, "Miniature coplanar implantable antenna on thin and flexible platform for fully wireless intracranial pressure monitoring system," Int. J. Antennas Propag., vol. 2017, pp. 1-9, Jan. 2017, doi: 10.1155/2017/9161083.

[14] N. Engheta, A. Alu, R. W. Ziolkowski, and A. Erentok, "Fundamentals of waveguides and antenna applications involving doublenegative (DNG) and single-negative (SNG) metamaterials," in Metamaterials: Physics and Engineering Explorations, N. Engheta and R. W. Ziolkowski, Eds. Piscataway, NJ, USA: IEEE Press, 2006, pp. 43-85.

[15] H. R. Stuart and A. Pidwerbetsky, "Electrically small antenna elements using negative permittivity resonators," IEEE Trans. Antennas Propag., vol. 54, no. 6, pp. 1644-1653, Jun. 2006.

[16] R. W. Ziolkowski, P. Jin, and C.-C. Lin, "Metamaterial-inspired engineering of antennas," Proc. IEEE, vol. 99, no. 10, pp. 1720-1731, Oct. 2011.

[17] K. B. Alici and E. Ozbay, "Electrically small split ring resonator antennas," J. Appl. Phys., vol. 101, no. 8, Apr. 2007, Art. no. 083104.

[18] P. Jin and R. W. Ziolkowski, "Multi-frequency, linear and circular polarized, metamaterial-inspired, near-field resonant parasitic antennas," IEEE Trans. Antennas Propag., vol. 59, no. 5, pp. 1446-1459, May 2011.

[19] C. G. M. Ryan and G. V. Eleftheriades, "Two compact, wideband, and decoupled meander-line antennas based on metamaterial concepts," IEEE Antennas Wireless Propag. Lett., vol. 11, pp. 1277-1280, 2012.

[20] S. Yan, P. J. Soh, and G. A. E. Vandenbosch, "Compact all-textile dual-band antenna loaded with metamaterial-inspired structure," IEEE Antennas Wireless Propag. Lett., vol. 14, pp. 1486-1489, 2015.
[21] S. Yan, X. Wang, Y. Hu, and G. A. E. Vandenbosch, "Lowprofile omnidirectional antenna for automatic dependent surveillanceBroadcast applications," Electron. Lett., vol. 51, no. 22, pp. 1732-1734, Oct. 2015.

[22] N. Zhu, R. W. Ziolkowski, and H. Xin, "Electrically small GPS L1 rectennas," IEEE Antennas Wireless Propag. Lett., vol. 10, pp. 935-938, 2011.

[23] H. Mirzaei and G. V. Eleftheriades, "A compact frequencyreconfigurable metamaterial-inspired antenna," IEEE Antennas Wireless Propag. Lett., vol. 10, pp. 1154-1157, 2011.

[24] G. Mumcu, K. Sertel, and J. L. Volakis, "Miniature antenna using printed coupled lines emulating degenerate band edge crystals," IEEE Trans. Antennas Propag., vol. 57, no. 6, pp. 1618-1624, Jun. 2009.

[25] J. L. Volakis and K. Sertel, "Narrowband and wideband metamaterial antennas based on degenerate band edge and magnetic photonic crystals," Proc. IEEE, vol. 99, no. 10, pp. 1732-1745, Oct. 2011.

[26] K. W. Leung, E. H. Lim, and X. S. Fang, "Dielectric resonator antennas: From the basic to the aesthetic," Proc. IEEE, vol. 100, no. 7, pp. 2181-2193, Jul. 2012.

[27] A. Petosa and A. Ittipiboon, "Dielectric resonator antennas: A historical review and the current state of the art," IEEE Antennas Propag. Mag., vol. 52, no. 5, pp. 91-116, Oct. 2010.

[28] See. [Online]. Available: https://www.johansontechnology.com/antennas

[29] E. Semouchkina, "Development of miniature microwave components by using high contrast dielectrics," in Microwave and Millimeter Wave Technologies From Photonic Bandgap Devices to Antenna Applications, I. Minin, Ed. Rijeka, Croatia: InTech, 2010, pp. 231-256.

[30] A. Hoorfar and A. Perrotta, "An experimental study of microstrip antennas on very high permittivity ceramic substrates and very small ground planes," IEEE Trans. Antennas Propag., vol. 49, no. 5, pp. 838-840, May 2001.

[31] T. K. Lo, C.-O. Ho, Y. Hwang, E. K. W. Lam, and B. Lee, "Miniature aperture-coupled microstrip antenna of very high permittivity," Electron. Lett., vol. 33, pp. 9-10, Jan. 1997.

[32] J. R. James and A. Henderson, "Electrically short monopole antennas with dielectric or ferrite coatings," Proc. Inst. Electr. Eng., vol. 125, no. 9 , p. 793,1978

[33] B. Lee and F. J. Harackiewicz, "Miniature microstrip antenna with a partially filled high-permittivity substrate," IEEE Trans. Antennas Propag., vol. 50, no. 8, pp. 1160-1162, Aug. 2002.

[34] E. Semouchkina, A. Baker, G. B. Semouchkin, M. Lanagan, and R. Mittra, "New approaches for designing microstrip filters utilizing mixed dielectrics," IEEE Trans. Microw. Theory Techn., vol. 53, no. 2, pp. 644-652, Feb. 2005.

[35] H. Jiang et al., "Miniaturized and reconfigurable CPW square-ring slot antenna loaded with ferroelectric BST thin film varactors," IEEE Trans. Antennas Propag., vol. 60, no. 7, pp. 3111-3119, Jul. 2012.

[36] V. A. Tamma, T.-E. Wang, E. F. Kuester, W. Park, C. L. Holloway, and J. Zhou, "Metamaterial inspired electrically small patch antenna," in Proc. IEEE Int. Symp. Antennas Propag. (APSURSI), Spokane, WA, USA, Jul. 2011, pp. 3-8, doi: 10.1109/APS.2011.5996387.

[37] Y. Yashchyshyn and J. W. Modelski, "Rigorous analysis and investigations of the scan antennas on a ferroelectric substrate," IEEE Trans. Microw. Theory Techn., vol. 53, no. 2, pp. 427-438, Feb. 2005.

[38] K. A. Jose, V. K. Varadan, and V. V. Varadan, "Experimental investigations on electronically tunable microstrip antennas," Microw. Opt. Technol. Lett., vol. 20, no. 3, pp. 166-169, Feb. 1999.

[39] S. Courreges et al., "Back-to-back tunable ferroelectric resonator filters on flexible organic substrates," IEEE Trans. Ultrason., Ferroelectr. Freq. Control, vol. 57, no. 6, pp. 1267-1275, Jun. 2010.

[40] T. H. Hand and S. A. Cummer, "Frequency tunable electromagnetic metamaterial using ferroelectric loaded split rings," J. Appl. Phys., vol. 103, no. 6, Mar. 2008, Art. no. 066105.

[41] M. Gil et al., "Tunable sub-wavelength resonators based on bariumstrontium-titanate thick-film technology," IET Microw., Antennas Propag., vol. 5, no. 3, pp. 316-323, 2011.

[42] E. Ozbay, K. Aydin, S. Butun, K. Kolodziejak, and D. Pawlak, "Ferroelectric based tuneable SRR based metamaterial for microwave applications," in Proc. 37th Eur. Microw. Conf., Munich, Germany, Oct. 2007, pp. 497-499.

[43] K. B. Alici, F. Bilotti, L. Vegni, and E. Ozbay, "Miniaturized negative permeability materials," Appl. Phys. Lett., vol. 91, no. 7, Aug. 2007, Art. no. 071121. 
[44] B. Moradi, U. Matrinez, and J. Garcia, "Split square resonators using high dielectric constant resonator," Electron. Lett., vol. 52, no. 21, pp. 1786-1787, Oct. 2016.

[45] C.-C. Lin, R. W. Ziolkowski, J. A. Nielsen, M. H. Tanielian, and C. L. Holloway, "An efficient, low profile, electrically small, threedimensional, very high frequency magnetic EZ antenna," Appl. Phys. Lett., vol. 96, no. 10, Mar. 2010, Art. no. 104102.

[46] C. Wang, M. Zhang, B. Stern, M. Lipson, and M. Lončar, "Nanophotonic lithium niobate electro-optic modulators," Opt. Express, vol. 26, no. 2, pp. 1547-1555, Jan. 2018.

[47] N. Dagli, High-Speed Photonic Devices. Boca Raton, FL, USA: CRC Press, 2007.

[48] E. L. Wooten et al., "A review of lithium niobate modulators for fiberoptic communications systems," IEEE J. Sel. Topics Quantum Electron., vol. 6, no. 1, pp. 69-82, Jan. 2000.

[49] W. S. T. Rowe and R. B. Waterhouse, "Efficient wideband printed antennas on lithium niobate for OEICs," IEEE Trans. Antennas Propag., vol. 51, no. 6, pp. 1413-1415, Jun. 2003.

[50] N. Kohmu, H. Murata, and Y. Okamura, "Electro-optic modulator using an antenna-coupled-electrode array and a polarization-reversed structure for a radar tracking system," Radio Sci. Bull., vol. 349, pp. 32-39, Jun. 2014.

[51] G. Lefort and T. Razban, "Microstrip antennas printed on lithium niobate substrate," Electron. Lett., vol. 33, no. 9, pp. 726-727, 1997.

[52] Y. N. Wijayanto, A. Kanno, T. Kawanishi, H. Murata, and Y. Okamura, "Z-cut $\mathrm{LiNbO}_{3}$ optical modulator using patch-antenna with orthogonalgaps for millimeter-wave radar applications," in Proc. Int. Topical Meeting Microw. Photon., 9th Asia-Pasific Microw. Photon. Conf., Sapporo, Japan, Oct. 2014, pp. 20-23.

[53] T.-H. Lee, P.-I. Wu, and C.-T. Lee, "Intergraded $\mathrm{LiNbO}_{3}$ electrooptical electromagnetic field sensor," Microw. Opt. Technol. Lett., vol. 49, no. 9 , pp. 2312-2314, 2007

[54] A. E. Serebryannikov, M. Gokkavas, F. T. Gundogdu, G. A. E. Vandenbosch, A. Vasylchenko, and E. Ozbay, "Ultraminiature dual-band antenna based on subwavelength resonators on $\mathrm{LiNbO}_{3}$ substrate," in Proc. 12th Eur. Conf. Antennas Propag. (EuCAP), London, U.K., 2018, pp. 8-13, Paper T03-1.1.

[55] See. [Online]. Available: https://www.3ds.com/products-services/ simulia/products/cst-studio-suite/

[56] G. Kiziltas, D. Psychoudakis, J. L. Volakis, and N. Kikuchi, “Topology design optimization of dielectric substrates for bandwidth improvement of a patch antenna," IEEE Trans. Antennas Propag., vol. 51, no. 10, pp. 2732-2743, Oct. 2003.

[57] P. W. Kolb, T. S. Salter, J. A. McGee, H. D. Drew, and W. J. Padilla, "Extreme subwavelength electric GHz metamaterials," J. Appl. Phys. vol. 110, no. 5, Sep. 2011, Art. no. 054906.

[58] W.-C. Chen, C. M. Bingham, K. M. Bingham, K. M. Mak, N. W. Caira, and W. J. Padilla, "Extremely subwavelength planar magnetic metamaterials," Phys. Rev. B, Condens. Matter, vol. 85, no. 20, 2012, Art. no. 201104

[59] Z. Sheng and V. V. Varadan, "Tuning the effective properties of metamaterials by changing the substrate properties," J. Appl. Phys., vol. 101, no. 1, Jan. 2007, Art. no. 014909.

[60] K. B. Alici, A. E. Serebryannikov, and E. Ozbay, "Radiation properties and coupling analysis of a metamaterial based, dual polarization, dual band, multiple split ring resonator antenna," J. Electromagn. Waves Appl., vol. 24, nos. 8-9, pp. 1183-1193, 2010.

[61] R. O. Ouedraogo and E. J. Rothwell, "Metamaterial inspired patch antenna miniaturization technique," in Proc. IEEE Antennas Propag. Soc. Int. Symp., Toronto, ON, Canada, Jul. 2010, pp. 11-17, doi: 10.1109/APS.2010.5561205.

[62] H. Odabasi, F. L. Teixeira, and D. O. Guney, "Electrically small, complementary electric-field-coupled resonator antennas," J. Appl. Phys., vol. 113, no. 8, Feb. 2013, Art. no. 084903.

[63] R. Samson Daniel, R. Pandeeswari, and S. Raghavan, "Offset-fed complementary split ring resonators loaded monopole antenna for multiband operations," AEU-Int. J. Electron. Commun., vol. 78, pp. 72-78, Aug. 2017.

[64] J. A. Harrington, Time-Harmonic Electromagnetic Fields. New York, NY, USA: McGraw-Hill, 1968.

[65] A. E. Serebryannikov, M. Mutlu, and E. Ozbay, "Dielectric inspired scaling of polarization conversion subwavelength resonances in open ultrathin chiral structures," Appl. Phys. Lett., vol. 107, no. 22, Nov. 2015, Art. no. 221907
[66] D. R. Bosomworth, "The far infrared optical properties of $\mathrm{LiNbO}_{3}$," Appl. Phys. Lett., vol. 9, no. 9, pp. 330-331, Nov. 1966.

[67] R. Harrington, "On the gain and beamwidth of directional antennas," IRE Trans. Antennas Propag., vol. 6, no. 3, pp. 219-225, Jul. 1958.

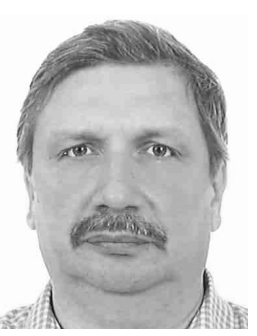

Andriy E. Serebryannikov (Senior Member, IEEE) received the Diploma degree in physics and technology from the Kharkiv Polytechnic Institute, Kharkiv, Ukraine, in 1990, and the Ph.D. degree in radio physics from the Kharkiv National University, Kharkiv, in 1996

He has been a Visiting Researcher with The University of Sydney, Sydney, NSW, Australia, Yokohama City University, Yokohama, Japan, and Bilkent University, Ankara, Turkey. In 2014, he joined the Faculty of Physics, Adam Mickiewicz University, Poznań, Poland. Since 2017, he has been a Research Fellow with the Katholieke Universiteit Leuven, Leuven, Belgium. He has published more than 100 journal articles, more than 50 conference papers, and three book chapters. His current research interests include theory and applications of metasurfaces, metamaterials, surface plasmons, and polarization manipulation.

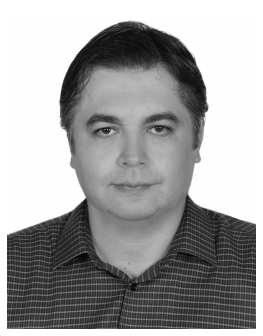

Mutlu Gokkavas received the B.Sc. and M.Sc. degrees in electrical engineering from Bilkent University, Ankara, Turkey, in 1994 and 1996, respectively, and the $\mathrm{Ph} . \mathrm{D}$. degree in electrical engineering from Boston University, Boston, MA, USA, in 2001 .

$\mathrm{He}$ is currently a Senior Research Associate with the Nanotechnology Research Center (NANOTAM), Bilkent University. His research interests include applications of $\mathrm{LiNbO}_{3}$ in different frequency ranges, chiral nanostructures, and solar cells.

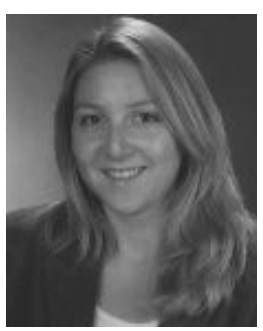

Tamara Funda Gundogdu received the B.Sc. and M.Sc. degrees in physics from Yildiz Technical University, Istanbul, Turkey, in 1998 and 2001, respectively, and the Ph.D. degree in physics/material science and technology from the University of Crete, Heraklion, Greece, in 2009.

She is currently a Senior Research Associate with the Nanotechnology Research Center (NANOTAM), Bilkent University, Ankara, Turkey. Her research interests include photonic crystals, subwavelength resonators, and metamaterials.

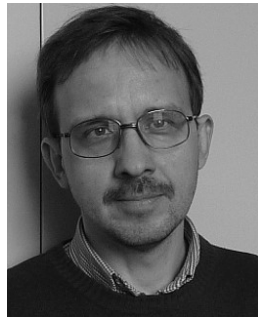

Vladimir Volski graduated from the Moscow Power Engineering Institute, Moscow, Russia, in 1987. $\mathrm{He}$ received the Candidate of Science (Ph.D.) degree from the Moscow Power Engineering Institute in 1993.

In 1987, he joined the Antennas and Propagation of Radio waves Division, Moscow Power Engineering Institute, as a Researcher. Since January 1996, he has been a Researcher with the ESAT-TELEMIC Division, Katholieke Universiteit Leuven, Leuven, Belgium. His main research interests include electromagnetic theory, computational electromagnetics, antenna design, and measuring of electromagnetic radiation. 


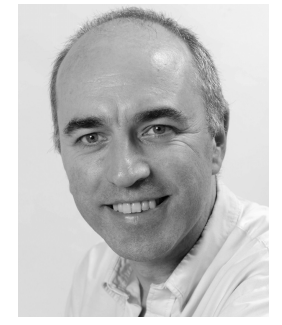

Guy A. E. Vandenbosch (Fellow, IEEE) received the M.S. and Ph.D. degrees in electrical engineering from the Katholieke Universiteit Leuven, Leuven, Belgium, in 1985 and 1991, respectively.

Since 1993, he has been a Lecturer with the Katholieke Universiteit Leuven, where he has also been a Full Professor, since 2005. He has taught or teaches courses on electromagnetic waves; antennas; electromagnetic compatibility (EMC); fundamentals of communication and information theory; electrical engineering, electronics, and electrical energy; and digital steering and measuring techniques in physics. From September 2014 to December 2014, he was a Visiting Professor with Tsinghua University, Beijing, China. His work has been published in approximately 340 articles in international journals and has led to about 390 presentations at international conferences. His research interests are in the areas of electromagnetic theory, computational electromagnetics, planar antennas and circuits, nanoelectromagnetics, EM radiation, EMC, and bio-electromagnetics.

Dr. Vandenbosch has been a member of the IEEE Electromagnetics Award Committee since 2017. From 1999 to 2004, he was the Vice-Chairman; from 2005 to 2009, he was the Secretary; and from 2010 to 2016, he was the Chairman of the IEEE Benelux Chapter on Antennas and Propagation. From 2002 to 2004, he was the Secretary of the IEEE Benelux Chapter on EMC. From 2012 to 2014, he was the Secretary of the Belgian National Committee for Radio-Electricity (URSI), where he is also in charge of the commission.

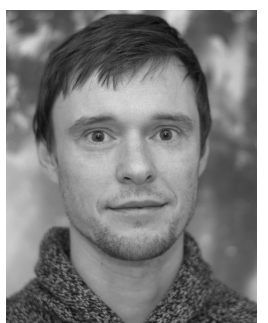

Alexander Vasylchenko received the Ph.D. degree in electrical engineering from the Interuniversity Microelectronics Centre (IMEC), Katholieke Universiteit Leuven, Leuven, Belgium, in 2010.

He was working for leading research and development centers in Europe with involvement in ICT industrial projects for global technology companies. Since 2012, he has been at the technical forefront in several IT startups such as Megion Research and Development GmbH, Vienna, Austria, Mycelium SA, Luxembourg, Luxembourg, and Grid Singularity GmbH, Vienna. In 2016, he founded Sofitto NV, Leuven, Belgium, a FinTech startup merging blockchain technology into conventional banking card products. He has written numerous articles on antenna and RF design and conducted talks and workshops on essential applications of blockchain technology in the financial and energy industries. He was a Guest Lecturer with the Howest University College, Bruges, Belgium, in 2018.

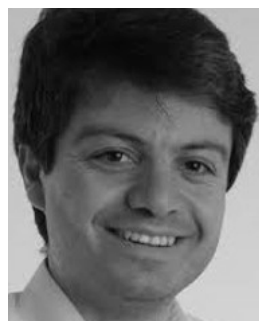

Ekmel Ozbay received the M.S. and Ph.D. degrees in electrical engineering from Stanford University, Stanford, CA, USA, in 1989 and 1992, respectively.

He worked as a Post-Doctoral Research Associate with Stanford University and as a Scientist with Iowa State University, Ames, IA, USA. In 1995, he joined Bilkent University, Ankara, Turkey, where he is currently a Full Professor with the Physics Department and the Electrical and Electronics Engineering Department. He worked as an Editor for Scientific Reports (Nature), Optics Letters, Photonics and Nanostructures: Fundamentals and Applications (PNFA), and the IEEE JOURNAL OF QUANTUM ELECTRONICS (JQE) journals. He has published more than 440 articles in SCI journals. His articles have received more than 14000 SCI citations with an SCI H-index of 56. He has given more than 155 invited talks in international conferences. He recently became the Chief Executive Officer (CEO) of AB-MicroNano Inc., Ankara, Turkey, a spinoff company. In 2003, he founded the Nanotechnology Research Center (NANOTAM), Bilkent University, where he leads a research group working on nanophotonics, metamaterials, nanoelectronics, GaN/AlGaN metal organic chemical vapor deposition (MOCVD) growth, and GaN-based devices.

Dr. Ozbay was a recipient of the Adolph Lomb Medal of OSA in 1997 and the 2005 European Union Descartes Science Award. 